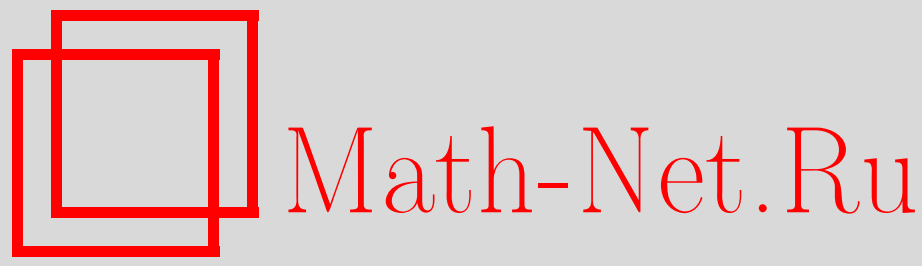

И. М. Дремин, Макроскопическая квантовая хромодинамика и кварк-глюонная плазма, ТМФ, 2012, том 170 , номер 1, 133-139

DOI: https://doi.org/10.4213/tmf6752

Использование Общероссийского математического портала Math-Net.Ru подразумевает, что вы прочитали и согласны с пользовательским соглашением http://www . mathnet.ru/rus/agreement

Параметры загрузки:

IP : 54.174 .149 .18

26 апреля 2023 г., 18:33:37

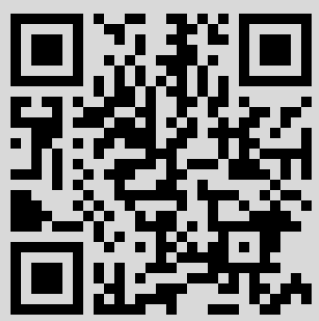




\title{
МАКРОСКОПИЧЕСКАЯ КВАНТОВАЯ ХРОМОДИНАМИКА И КВАРК-ГЛЮОННАЯ ПЛАЗМА
}

\begin{abstract}
Предложены уравнения макроскопической квантовой хромодинамики. Рассмотрены свойства кварк-глюонной среды при высоких энергиях, описываемые этими уравнениями. Проходящие через среду партонные токи приводят к испусканию черенковских глюонов, эффекту кильватерной струи и переходному излучению. Сравнение с экспериментом говорит о довольно больших значениях хромопроницаемости этой среды. Из анализа дисперсионных уравнений следует, что собственные моды среды неустойчивы.
\end{abstract}

Ключевые слова: квантовая хромодинамика, кварк, глюон, хромопроницаемость.

Экспериментальные данные наглядно свидетельствуют о том, что частицы, рожденные в соударениях тяжелых ионов при высоких энергиях, нельзя описывать как возникающие в результате независимых взаимодействий отдельных нуклонов. Кварк-глюонная среда, образующаяся при столкновениях высокоэнергичных ядер, несомненно обладает некими коллективными свойствами (см. обзорную статью [1]). В классическом приближении уравнения КХД оказываются подобными уравнениям КЭД, поэтому представляется вполне естественным использовать аналогию с электродинамическими процессами в обычном веществе. Изучение энергетических потерь партонов, проходящих через такую среду, является наиболее эффективным способом исследования отклика среды на их воздействие.

Существуют два подхода к решению этой проблемы. Уравнения КХД в вакууме применяются для изучения воздействия на проходящий партон внешних полей полей кварков и глюонов, составляющих среду. В них происходят такие процессы, как рассеяние, тормозное излучение и синхротронное излучение партонов, т. е. процессы, в которых вектор скорости излучающего партона претерпевает заметные изменения. Воздействие среды приводит к эффекту Тер-Микаеляна и потери энергии за счет эффекта Ландау-Померанчука-Мигдала. При другом подходе рассматриваются коллективные отклики среды путем введения феноменологического понятия проводимости $\epsilon$, которое учитывает наличие внутренних токов. При этом вектор

* Физический институт им. П. Н. Лебедева РАН, Москва, Россия. E-mail: dremin@td.lpi.ru 
скорости начального партона (тока) практически остается неизменным, а весь эффект возникает за счет поляризации среды, описываемой выражением

$$
\mathbf{P}=\frac{\epsilon-1}{4 \pi} \mathbf{E} .
$$

Таким образом, уравнения КХД в среде, предложенные впервые в работе [2], отличаются от соответствующих уравнений в вакууме наличием хромопроницаемости $\epsilon$ кварк-глюонной среды (ее коллективного отклика). Они предсказывают такие эффекты, как излучение черенковских глюонов, кильватерная струя и переходное излучение. Именно этот подход рассматривается в настоящей работе.

Малость изменения скорости не означает, что соответствующие потери энергии малы. В случае ультрарелятивистских частиц $(\gamma \gg 1)$ относительное изменение скорости оказывается намного меньше, чем относительная потеря энергии, поскольку они связаны выражением

$$
\frac{\Delta E}{E}=\left(\gamma^{2}-1\right) \frac{\Delta v}{v},
$$

из которого наглядно следует сделанное утверждение.

По аналогии с электродинамикой учет среды сводится к тому, что в $F^{\mu \nu}$ вектор $\mathbf{E}$ заменяется на $\mathbf{D}=\epsilon \mathbf{E}$. Далее можно получить лагранжиан КХД в среде и вывести уравнения для напряженностей полей и потенциалов (детали вывода см. в работе [2]). В действительности для наших целей достаточно ограничиться глюодинамикой, потому что глюоны являются основной активной компонентой структурных функций, проявляющей себя в процессах при высоких энергиях. В терминах потенциалов уравнения глюодинамики в среде принимают вид [2]

$$
\begin{aligned}
\Delta \mathbf{A}_{a}-\epsilon \frac{\partial^{2} \mathbf{A}_{a}}{\partial t^{2}}= & -\mathbf{j}_{a}-g f_{a b c}\left(\frac{1}{2} \operatorname{curl}\left[\mathbf{A}_{b} \mathbf{A}_{c}\right]+\epsilon \frac{\partial}{\partial t}\left(\mathbf{A}_{b} \Phi_{c}\right)+\frac{1}{2}\left[\mathbf{A}_{b} \operatorname{curl} \mathbf{A}_{c}\right]-\right. \\
& -\epsilon \Phi_{b} \frac{\partial \mathbf{A}_{c}}{\partial t}-\epsilon \Phi_{b} \operatorname{grad} \Phi_{c}- \\
& \left.-\frac{1}{2} g f_{c m n}\left[\mathbf{A}_{b}\left[\mathbf{A}_{m} \mathbf{A}_{n}\right]\right]+g \epsilon f_{c m n} \Phi_{b} \mathbf{A}_{m} \Phi_{n}\right) \\
\Delta \Phi_{a}-\epsilon \frac{\partial^{2} \Phi_{a}}{\partial t^{2}}= & -\frac{\rho_{a}}{\epsilon}+g f_{a b c}\left(-2 \mathbf{A}_{c} \operatorname{grad} \Phi_{b}+\mathbf{A}_{b} \frac{\partial \mathbf{A}_{c}}{\partial t}-\epsilon \frac{\partial \Phi_{b}}{\partial t} \Phi_{c}\right)+ \\
& +g^{2} f_{a m n} f_{n l b} \mathbf{A}_{m} \mathbf{A}_{l} \Phi_{b} .
\end{aligned}
$$

Классические уравнения получаются из них, если отбросить все члены с явно присутствующей константой связи $g$. При этом они становятся похожими на соответствующие уравнения КЭД. Однако следует иметь в виду, что $\epsilon$ зависит от $g$, и тем самым учитываются некоторые нелинейные эффекты. Более того, наличие цвета в сочетании с явными нелинейными членами в этих уравнениях приводит к такому неабелевому эффекту, как цветовая радуга, который мы не рассматриваем в настоящей работе.

Мы исследуем лишь классическую часть приведенных уравнений. Если рассмотреть классический ток со скоростью $\mathbf{v}$ вдоль оси $z$,

$$
\mathbf{j}(\mathbf{r}, t)=\mathbf{v} \rho(\mathbf{r}, t)=4 \pi g \mathbf{v} \delta(\mathbf{r}-\mathbf{v} t),
$$


то классическое решение в низшем порядке по константе связи для глюодинамики в среде будет иметь вид [2]

$$
\begin{aligned}
\Phi^{(1)}(\mathbf{r}, t) & =\frac{2 g}{\epsilon} \frac{\theta\left(v t-z-r_{\perp} \sqrt{\epsilon v^{2}-1}\right)}{\sqrt{(v t-z)^{2}-r_{\perp}^{2}\left(\epsilon v^{2}-1\right)}}, \\
\mathbf{A}^{(1)}(\mathbf{r}, t) & =\epsilon \mathbf{v} \Phi^{(1)}(\mathbf{r}, t)
\end{aligned}
$$

где индекс (1) указывает на решение порядка $g, r_{\perp}=\sqrt{x^{2}+y^{2}}-$ цилиндрическая координата, $z$ - направление оси симметрии. Это решение описывает излучение черенковских глюонов под характерным для него углом $\theta$ таким, что

$$
\cos \theta=\frac{1}{v \sqrt{\epsilon}}
$$

В случае постоянного $\epsilon>1$ этот угол строго фиксирован.

Впервые событие с такими свойствами было обнаружено при изучении сильных взаимодействий частиц в космических лучах [3]-[5]. Впоследствии данный эффект нашел серьезное подтверждение в экспериментах на ускорителе RHIC, в которых была обнаружена двугорбая структура вокруг партона (струи), проходящего через кварк-глюонную среду. В случае поглощающих сред хромопроницаемость $\epsilon$ имеет мнимую часть. Резкий фронт ударной волны (2) размывается, и угловое распределение черенковского излучения становится более широким, а $\delta$-функция в точке $\theta$, определяемой уравнением (4), заменяется на распределение типа брейт-вигнеровского (по углу) [6], [7] с максимумом примерно при том же значении угла (при замене $\epsilon$ на $|\epsilon|$ ) и шириной, пропорциональной мнимой части хромопроницаемости. Как и в электродинамике [6], нетрудно получить соответствующее выражение для распределения глюонов по энергиям и углам [7] на единичной длине пройденного пути:

$$
\frac{d N^{(1)}}{d \Omega d \omega}=\frac{\alpha_{\mathrm{S}} C \sqrt{x}}{2 \pi^{2}}\left(\frac{(1-x) \Gamma_{\mathrm{t}}}{\left(x-x_{0}\right)^{2}+\left(\Gamma_{\mathrm{t}}\right)^{2} / 4}+\frac{\Gamma_{\ell}}{x}\right),
$$

где

$$
x=\cos ^{2} \theta, \quad x_{0}=\frac{\epsilon_{1 \mathrm{t}}}{\left|\epsilon_{\mathrm{t}}\right|^{2} v^{2}}, \quad \Gamma_{j}=2 \frac{\epsilon_{2 j}}{\left|\epsilon_{j}\right|^{2} v^{2}}, \quad \epsilon_{j}=\epsilon_{1 j}+i \epsilon_{2 j}, \quad j=\mathrm{t}, \ell,
$$

$\epsilon_{1}-$ вещественная и $\epsilon_{2}$ - мнимая части хромопроницаемости $\epsilon$. Угол $\theta$ обозначает полярный угол по отношению к направлению оси струи, вызванной проходящим через среду партоном, которое принято здесь за ось z. Фактически он совпадает с углом, который задан уравнением (4), если учитывать только поперечную часть хромопроницаемости, положив в последних равенствах $\epsilon=\epsilon_{\mathrm{t}}$. Из уравнения (5) ясно видно, что поперечная $\epsilon_{\mathrm{t}}$ и продольная $\epsilon_{\ell}$ части хромопроницаемости несут ответственность за существенно различные эффекты. Кольцевая черенковская структура (коническое излучение) вокруг оси $z$ наглядно видна в первом слагаемом в правой части (5). Его вклад можно наблюдать даже при нулевой мнимой части хромопроницаемости $\epsilon$. Второй член определяется продольной составляющей хромопроницаемости $\epsilon$ и связан с эффектом кильватерной струи. Он исчезает при вещественных значениях $\epsilon$. Эти результаты отличаются от того, что получается в гидродинамике. 
Кольцевое распределение частиц вокруг струи, летящей в обратном направлении через кварк-глюонную среду, наблюдалось в виде двух максимумов (горбов) после проекции на диаметр кольца. Это полностью аналогично тому, что делал Черенков в своих оригинальных публикациях. Именно такое распределение было использовано нами [7] для сопоставления данных экспериментов на ускорителе RHIC с теоретическими формулами. При описании двухчастичных корреляций, измеренных коллаборациями STAR и PHENIX, было найдено [7], что вещественная и мнимая части хромопроницаемости $\epsilon=\epsilon_{1}+i \epsilon_{2}$ оказались соответственно равными 6 и 0.8 , т. е. вещественная часть значительно больше мнимой. Малость мнимой части отвечает малому поглощению, что способствует наблюдению этого эффекта. Вещественная часть задает расположение пиков, тогда как мнимая часть определяет ширину распределения вокруг них.

Вещественная часть хромопроницаемости может быть выражена через вещественную часть $\operatorname{Re} F_{0}(\omega)$ амплитуды упругого рассеяния вперед рассеиваемых квантов:

$$
\operatorname{Re} \Delta \epsilon=\operatorname{Re} \epsilon(\omega)-1=\frac{4 \pi N_{\mathrm{s}} \operatorname{Re} F_{0}(\omega)}{\omega^{2}}=\frac{N_{\mathrm{s}} \sigma(\omega) \rho(\omega)}{\omega}
$$

с учетом оптической теоремы, записанной в виде $\operatorname{Im} F_{0}(\omega)=(\omega / 4 \pi) \sigma(\omega)$. Здесь $\omega$ обозначает энергию кванта, $N_{\mathrm{s}}$ - плотность рассеивающих центров, $\sigma(\omega)$ - полное сечение и $\rho(\omega)$ - отношение вещественной и мнимой частей амплитуды $F_{0}(\omega)$ упругого рассеяния вперед. Таким образом, излучение черенковских глюонов возможно только в процессах с положительными $\operatorname{Re} F_{0}(\omega)$ или $\rho(\omega)$. K сожалению, в настоящий момент мы не можем теоретически вычислить эти характеристики непосредственно для глюонных амплитуд и вынуждены обращаться к их возможным аналогиям с адронными амплитудами, имея в виду, что глюоны играют наиболее активную роль во взаимодействиях адронов. Данные экспериментов с адронами подсказывают нам, что необходимое условие $\operatorname{Re} F_{0}(\omega)>0$ черенковского испускания глюонов могло бы быть выполнено по крайней мере в двух энергетических интервалах - в области резонансов (в их левых крыльях) и при весьма высоких энергиях.

Первая область проявляет себя при сравнительно низких энергиях вторичных частиц, регистрируемых в экспериментах на ускорителях SPS и RHIC. При малых масcax $\operatorname{Re} F_{0}(\omega)$ всегда положительна (т. е. $\epsilon>1$ ) в левых крыльях брейт-вигнеровских резонансов, поэтому черенковские глюоны могут излучаться в этих энергетических интервалах. Асимметрия распределения по массам $\rho$-мезонов, наблюдаемая в их лептонных модах распадов при прохождении их через среду при ядерных соударениях на ускорителе SPS [8], была объяснена именно испусканием низкоэнергичных черенковских глюонов [9], [10] с массами, соответствующими левому (низкоэнергичному) крылу брейт-вигнеровского резонанса. Более того, предсказывают, что это свойство должно быть общим для всех резонансов, проходящих через ядерную среду. Уже имеются некоторые предварительные экспериментальные указания в пользу такого заключения о многих других резонансах [9].

В обсуждавшихся выше экспериментах коллабораций STAR и PHENIX на ускорителе RHIC по изучению двух- и трехчастичных азимутальных корреляцияй в центральных соударениях тяжелых ионов также имеют дело со вторичными частицами с относительно малыми энергиями. Вместе с тем при этом наблюдался и новый эффект сдвига положений максимумов в полуцентральных ядерных соударениях [11]. 
Он был объяснен как проявление излучения от кильватерной струи, при этом использовались те же значения хромопроницаемости $\epsilon$ [12]. Было проведено также рассмотрение возможного переходного излучения на границе областей с конфайнментом и деконфайнментом [13].

Наше последующее изложение будет, наоборот, сконцентрировано на области очень высоких энергий. Мы изучаем свойства кварк-глюонной среды в рамках модели, в которой хромопроницаемость выше некоторого энергетического порога $\omega_{\mathrm{thr}}$ ведет себя как

$$
\operatorname{Re} \epsilon=1+\frac{\omega_{0}^{2}}{\omega^{2}}
$$

где $\omega_{0}$ является неким свободным вещественным параметром. Классические уравнения, получающиеся из уравнений (1), будучи переписаны в импульсном представлении, имеют решения, если выполнено следующее дисперсионное соотношение:

$$
\operatorname{det}(\omega, \mathbf{k}) \equiv\left|k^{2} \delta_{i j}-k_{i} k_{j}-\omega^{2} \epsilon_{i j}\right|=0,
$$

где индексы $i, j$ указывают на пространственные компоненты импульсов и хромопроницаемости. Написанные уравнения являются уравнениями шестого порядка по размерности импульсов. Однако члены шестого порядка сокращаются, и уравнение (8) приводится к двум уравнениям второго порядка

$$
\begin{gathered}
k^{2}-\omega^{2}-\omega_{0}^{2}=0 \\
\left(k^{2}-\omega^{2}-\omega_{0}^{2}\right)\left(1+\frac{\omega_{0}^{2}}{\omega^{2}}\right)-\frac{\omega_{0}^{4} k_{\mathrm{t}}^{2}}{\omega^{2}\left(\omega-k_{z}\right)^{2} \gamma}=0
\end{gathered}
$$

которые определяют внутренние моды возбуждений среды и распространение потока партонов через среду. Уравнение (9) явно показывает, что кварк-глюонная среда неустойчива, поскольку существует ветвь возбуждений с $\operatorname{Im} \omega>0$ для мод $k^{2}<\omega_{0}^{2}$. Таким образом, мы установили непосредственную связь универсального свойства роста полных сечений адронных взаимодействий при возрастании энергии с нестабильностью кварк-глюонной среды, используя положительность $\operatorname{Re} F_{0}(\omega)$ при высоких энергиях.

Уравнение (10) обладает решениями, ответственными за излучение черенковских глюонов налетающим пучком партонов и характеризуемых последним слагаемым в (10). Эти решения можно найти при $\omega=k_{z}+\delta, \delta \ll \omega$. При $k_{\mathrm{t}}=\omega_{0}$ и при $k_{\mathrm{t}} \neq \omega_{0}$ получаем соответственно решения с

$$
\begin{aligned}
\operatorname{Im} \delta_{1} & =\frac{\omega_{0}^{2}}{2 k_{z}\left[2 \gamma\left(1+\omega_{0}^{2} / k_{z}^{2}\right)\right]^{1 / 3}}, \\
\operatorname{Im} \delta_{2} & =\frac{\omega_{0}^{2} k_{\mathrm{t}}}{k_{z}\left[\gamma\left|k_{\mathrm{t}}^{2}-\omega_{0}^{2}\right|\left(1+\omega_{0}^{2} / k_{z}^{2}\right)\right]^{1 / 2}} .
\end{aligned}
$$

Решения дисперсионного уравнения (8) определяют функцию Грина рассматриваемой системы:

$$
G(t, z)=\frac{1}{2 \pi^{2}} \int_{-\infty}^{\infty} d k \int_{C(\omega)} \frac{1}{\operatorname{det}(\omega, \mathbf{k})} e^{-i \omega t+i k z} d \omega
$$


где контур $C(\omega)$ проходит выше всех сингулярностей подынтегрального выражения. Поэтому положительность $\operatorname{Im} \omega$, найденная в уравнениях (10), определяет абсолютную неустойчивость системы. Заметим, что неустойчивость при $k_{\mathrm{t}}=\omega_{0}$ оказывается сильнее неустойчивости при $k_{\mathrm{t}} \neq \omega_{0}$ приблизительно в $\gamma^{1 / 6}$ раз (этот множитель примерно вчетверо больше в экспериментах на LHC по сравнению с RHIC). Показатель экспоненты уменьшается асимптотически по закону $\gamma^{-1 / 3}$ и оказывается примерно в 16 раз меньше в экспериментах на LHC по сравнению с RHIC. При этом оказывается, что черенковские глюоны испускаются с постоянным поперечным импульсом $k_{\mathrm{t}}=\omega_{0}$, и их число пропорционально $d \omega / \omega^{2} \cdot \theta\left(\omega-\omega_{\mathrm{thr}}\right)$ для хромопроницаемости $\epsilon(\omega)$, заданной выражением (7) с учетом порогового значения энергий, при которых оно только и справедливо. Это отличается от традиционных утверждений о постоянстве угла черенковского излучения и числа глюонов $\propto d \omega$ (или полной потери энергии, пропорциональной $\omega d \omega)$, которые справедливы только для постоянной хромопроницаемости.

В заключение отметим, что уравнения КХД в среде предсказывают излучение черенковских глюонов и появление событий с кольцевой структурой расположения вторичных частиц. Экспериментальные данные, полученные на ускорителе RHIC, позволяют найти как вещественную (довольно большую), так и мнимую (относительно малую) части хромопроницаемости. Большая вещественная часть указывает на высокую плотность (большие $N_{\mathrm{s}}$ в $\left.(6)\right)$ кварк-глюонной плазмы, а малая мнимая часть способствует более свободному прохождению партонов и, следовательно, экспериментальному обнаружению эффекта.

Другим экспериментально найденным фактом, связанным с черенковскими глюонами, является асимметрия форм резонансных пиков для всех резонансов, проходящих через кварк-глюонную среду. Это наглядно видно при изучении двухлептонных каналов их распадов (на SPS и некоторых других ускорителях).

Помимо черенковских глюонов, имеется также классический коллективный эффект излучения от кильватерной струи, образующейся в хвосте движения проходящего через среду партона. Этот эффект наблюдался в эксперименте как сдвиг положений максимумов в двугорбых распределениях при полуцентральных соударениях тяжелых ионов на ускорителе RHIC. Происхождение этого смещения максимумов приписывается специфической направленности излучения от кильватерной струи позади партона, напоминающего дипольное излучение. Кроме того, возможно и появление переходного излучения.

Относительно обнаружения новых эффектов на LHC можно ожидать появления черенковских глюонов, испущенных движущимися вперед партонами с очень высокими энергиями в экспериментах без использования специальных триггеров. Здесь значения хромопроницаемости могут быть близкими к единице (на это указывают данные, полученные в космических лучах). Законы дисперсии кварк-глюонной среды при высоких энергиях отличаются от тех, с которыми мы привыкли иметь дело в электромагнетизме (закон Ленгмюра). Основное отличие состоит в том, что хромопроницаемость может превышать единицу. Нами рассмотрена конкретная теоретическая модель с такими свойствами. Дисперсионные уравнения глюодинамики в среде показывают, что кварк-глюонная среда неустойчива и реагирует на внешние проходящие через нее партоны с высокими энергиями путем рождения черенковских глюонов со специфическими характеристиками. 
Благодарности. Работа была поддержана РФФИ (гранты № 09-02-00741, 08-0291000-ЦЕРН) и Программой РАН-ЦЕРН.

\section{Список литературы}

[1] И. М. Дремин, А. В. Леонидов, УФН, 180 (2010), 1167-1196.

[2] I. M. Dremin, Eur. Phys. J. C, 56:1 (2008), 81-86, arXiv: 0802.4022.

[3] А. В. Апанасенко, Н. А. Добротин, И. М. Дремин, К. А. Котельников, Писъма в ЖЭТФ, 30:2 (1979), 157-161.

[4] И. М. Дремин, Писъма в ЖЭТФ, 30:2 (1979), 152-156; Исправление, 30:2 (1979), 638.

[5] И. М. Дремин, ЯФ, 33:5 (1981), 1357-1368.

[6] V. M. Grichine, Nucl. Instr. Meth. Phys. Res. A, 482:3 (2002), 629-633.

[7] I. M. Dremin, M. R. Kirakosyan, A. V. Leonidov, A. V. Vinogradov, Nucl. Phys. A, 826:1-2 (2009), 190-197, arXiv: 0809.2472.

[8] S. Damjanovic et al. (NA60 Collab.), Phys. Rev. Lett., 96:16 (2006), 162302, 5 pp.

[9] I. M. Dremin, V. A. Nechitailo, Internat. J. Modern Phys. A, 24:6 (2009), 1221-1228.

[10] I. M. Dremin, Nucl. Phys. A, 767 (2006), 233-247, arXiv: hep-ph/0507167.

[11] W. G. Holzmann et al. (PHENIX Collab.), Nucl. Phys. A, 830:1-4 (2009), 781-784, arXiv: 0907.4833.

[12] I. M. Dremin, Mod. Phys. Lett. A, 25:8 (2010), 591-595, arXiv: 0911.3233.

[13] I. M. Dremin, Some remarks about the transition gluon radiation, arXiv: 1003.2145. 